\title{
An evaluation of dual source computed tomography used with the de Weert classification to detect vulnerable plaque, using IVUS virtual histology as a standard of reference
}

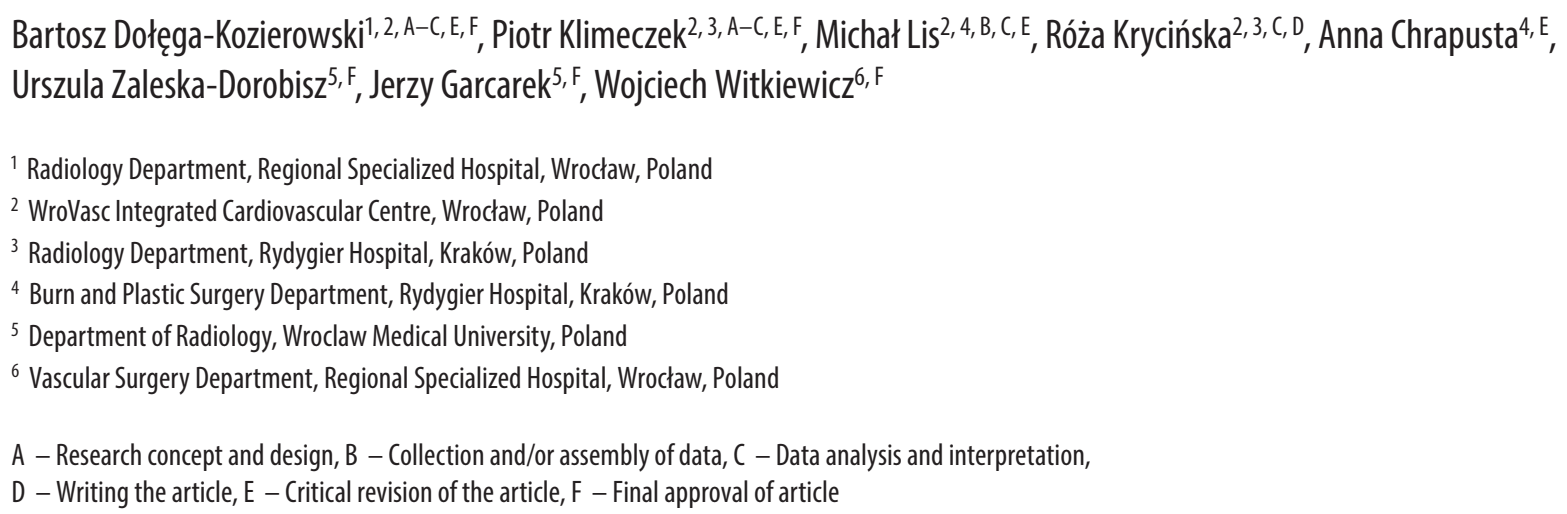

Address for correspondence

\section{Michał Lis}

E-mail: lis.michal.md@gmail.com

\section{Funding sources}

This publication is part of the WroVasc Integrated Cardiovascular Centre Project, $c 0$-financed by the European Regional Development Fund, within the 2007-2013 Innovative Economy Operational Program, carried out at the Provincial Specialized Hospital, Research and Development Center in Wroclaw: "European Funds - for the development of innovative economy".

Conflict of interest

none declared

Received on May 18, 2016

Revised on September 21, 2016

Accepted on October 26, 2016

DOI

DOI: $10.17219 /$ acem/66365

\section{Copyright}

Copyright by Author(s)

This is an article distributed under the terms of the

Creative Commons Attribution Non-Commercial License

(http://creativecommons.org/licenses/by-nc-nd/4.0/)

\section{Abstract}

Background. One of the main risk factors for cerebral ischemic events is atherosclerotic disease of the internal carotid artery (ICA). Nowadays, increasing attention is being paid to the relationship between the morphological features of atherosclerotic plaque and the occurrence of stroke. Several studies have demonstrated that the presence of specific vulnerable plaque types, with a large lipid core and thin fibrous cap, can be used as an independent risk predictor of cerebral ischemic events.

Objectives. The present study is an attempt to develop the method of plaque surface morphology assessment presented by de Weert et al. by correlating the results of Dual Source Computed Tomography (DSCT) with those from intravascular ultrasound virtual histology (IVUS-VH)

Material and methods. A group of 30 symptomatic patients (13 men and 17 women; $72 \pm 9$ years) with ICA stenosis suspected on the basis of ultrasound imaging (US) and confirmed to be above $70 \%$ in DSCT underwent intravascular ultrasound (IVUS) imaging.

Results. The results of DSCT were categorized according to the de Weert classification. There were 13 cases (43\%) with smooth wall surfaces, 10 cases (33\%) with discreet wall irregularities, and seven cases (23\%) with incursions of contrast, indicating the presence of ulceration. In the IVUS-VH examinations, 4 out of 30 cases (13\%) were identified as having adaptive intimal thickening (AIT), 4 (13\%) as showing pathological intimal thickening (PIT), 6 (20\%) with fibroatheromas (FA), six (20\%) with fibrocalcific plaque (FCa), and 10 (33\%) as having thin-cap fibroatheroma (TCFA), which is high-risk plaque. Comparing the above results showed that all the patients with confirmed wall ulceration in DSCT were characterized as having high-risk plaque in IVUS-VH.

Conclusions. Using DSCT with the de Weert classification of plaque surface morphology makes reliable detection of ulcerations possible; therefore, this could become a significant new technique to improve current imaging protocols for patients with a high risk of ischemic cerebrovascular events.

Key words: atherosclerosis, ischemic stroke, IVUS, DSCT, ICA stenosis 
Ischemic stroke is one of the most common causes of death and long-term disability in industrialized countries. Although stroke rates adjusted for age have continued a downward trend over the past three decades, the aging population could cause the number of strokes to increase in the near future. ${ }^{1}$

One of the main risk factors for ischemic stroke is atherosclerotic disease of the internal carotid artery (ICA). For this reason, evaluation of the degree of ICA stenosis has become part of the currently used algorithms for choosing optimal treatment methods. The beneficial effects of intervention in patients with stenosis above $70 \%$ have been identified in two large randomized studies: The North American Symptomatic Carotid Endarterectomy Trial (NASCET) and the Medical Research Council's European Carotid Surgery Trial (ECST). ${ }^{2-4}$ On the other hand, for over a decade more and more attention has been drawn to the relationship between the morphological features of atherosclerotic plaque and the occurrence of ischemic events, particularly with regard to acute coronary syndrome (ACS). ${ }^{5}$ The development of novel imaging methods has made it possible to identify in vivo the presence of plaque ruptures and plaque hemorrhages, as well as assess the size of a lipid or necrotic core and the thickness of a fibrous tissue cap. Based on these factors, the concept of "vulnerable plaque" was introduced, referring to lesions with a high tendency to rupture, resulting in embolization or thrombosis. ${ }^{6}$

Several studies have demonstrated that the presence of a specific vulnerable plaque type with a large lipid core and a thin fibrous cap can be used as an independent risk predictor of ischemic cerebrovascular events. ${ }^{7,8}$ Therefore, it seems that in the coming years diagnostic algorithms should be augmented with assessments of atherosclerotic plaque morphology. ${ }^{9-11}$

In 2009, in a study published in Stroke, de Weert et al. proposed an interesting method for indirect assessment of plaque morphology using minimally invasive multidetector computed tomography angiography (MDCTA) and their own classification system. ${ }^{12}$ The authors correlated the MDCTA results with clinical data regarding the severity of stenosis, cardiovascular risk factors and symptomatology.

The imaging protocol for intravascular ultrasound virtual histology (IVUS-VH) has introduced a new level of quality to the assessment of lesion morphology that makes it possible to put histopathological knowledge to direct use in in vivo intravascular diagnosis. ${ }^{13}$ The present study is an attempt to develop the method presented by de Weert et al. by correlating the results of Dual Source Computed Tomography (DSCT) with those obtained from IVUS-VH.

\section{Material and methods}

From January 2011 to June 2013, a group of 170 patients with ICA stenosis found in ultrasound imaging (US) un- derwent dual source computed tomography (DSCT) to confirm the presence of significant stenosis (above 70\%) in the ostium of the ICA. On this basis, the patients were included in the intravascular ultrasound (IVUS) study.

The IVUS study inclusion criteria were: (1) ostial ICA stenosis above $70 \%$ in CT according to the NASCET criteria; (2) patency of the contralateral carotid artery; (3) no kinking, coiling, tortuosity, hypoplasty or occlusion of the ICA; (4) age below 81 years; (5) no contraindications for an embolic protection device; (6) a plaque-free section of the ICA $25 \mathrm{~mm}$ above the ostium; (7) no decompensated heart failure (NYHA III-IV). Hence, the final study group consisted of 30 patients (13 men and 17 women; $72 \pm$ 9 years) with multiple cardiovascular risk factors (Tables 1 and 2).

The experimental protocol and informed consent procedure complied with the Helsinki Convention and were approved by a bioethics commission.

Scanning was performed on a 64-slice dual source CT scanner (Somatom Definition, Siemens Medical Solutions, Forchheim, Germany) according to Persson's Dual Energy protocol using $140 \mathrm{kV}$ and $80 \mathrm{kV}$ lamps. ${ }^{14}$ When an $80 \mathrm{kV}$ lamp is used, both contrast and calcifications are characterized by high density. When a $140 \mathrm{kV}$ lamp is used, calcifications can be differentiated and removed from the image due to their higher density. The DSCT scan range extended from the aortic arch to the circle of Willis. All the patients received $50 \mathrm{~mL}$ of contrast material (400 mg/mL Iomeron 400, Bracco Imaging Deutschland $\mathrm{GmbH}$, Konstanz, Germany), followed by $60 \mathrm{~mL}$ of saline bolus chaser, both with an injection rate of $6 \mathrm{~mL} / \mathrm{s}$. Synchronization between the passage of the contrast ma-

Table 1. Inclusion and exclusion criteria for the study

\begin{tabular}{|l|l|}
\hline Inclusion criteria & Exclusion criteria \\
\hline $\begin{array}{ll}\text { Ostial ICA stenosis }>70 \% \text { in CT } \\
\text { according to NASCET Criteria }\end{array}$ & inappropriate anatomy (kinking, \\
coiling) \\
Symptomatic stenosis & difficulties in setting neuroprotection \\
Age $<81$ years old & NYHA III, IV \\
Patency of contralateral artery & ICA hypoplasty or occlusion \\
\hline
\end{tabular}

Table 2. Demographic and epidemiological data of the study group

\begin{tabular}{|l|c|}
\hline Number of patients & 30 \\
\hline Age (years) & $72 \pm 9$ \\
Obesity $(n)$ & 5 \\
Hypertension $(n)$ & 19 \\
Diabetes $(n)$ & 15 \\
Stroke $(n)$ & 12 \\
Hyperlipidemia $(n)$ & 30 \\
Coronary Disease $(n)$ & 8 \\
\hline
\end{tabular}


terial and data acquisition was achieved by real-time bolus tracking at the level of the ascending aorta. The trigger threshold was set at an increase in attenuation of 110 Hounsfield units above baseline attenuation.

The DSCT images were processed on a Syngo-Via workstation (Siemens Medical Solutions, Forchheim, Germany) using dedicated 3-dimensional analysis software with hard plaque and head bone removal algorithms. Carotid arteries were evaluated with multiplanar reformatting (MPR) software, adjusting oblique planes to assess them in multiple short and long axes.

The surface of a lesion was evaluated and classified according to the de Weert classification into one of the three types: (1) smooth, (2) irregular and (3) ulcerated. Atherosclerotic plaque was classified as ulcerated when the contrast material was observed in the surrounding plaque, beyond the vascular lumen. Plaque classified as irregular showed discreet irregularities within the vessel walls, with an absence of ulcerations. Lesions without any ulcerations or distortions of lumen contour were classified as smooth.

In all the patients undergoing the IVUS procedure, embolic protection devices (Emboshield NAV6 Device, Abbott Vascular, Abbott Park, IL, USA) were used. Every examination was carried out by the same team, with a similar transducer position: $30 \mathrm{~mm}$ from the common carotid artery bifurcation. The images were obtained using $20 \mathrm{MHz}$ transducers, a 3.2-Fr IVUS catheter (Eagle Eye Gold, Volcano Corporation, Rancho Cordova, USA) with the options: gray scale, chroma flow and virtual histology $(\mathrm{VH})$ protocol. All the analyses were performed at an independent external core laboratory (Kraków Cardiovascular Research Institute, Krakow, Poland).

Internal carotid artery lesions observed with IVUS were classified into 5 categories ${ }^{15,16}$ :

- Adaptive intimal thickening (AIT): plaque comprised of mainly fibrous tissue; calcification, fibrofatty and/or necrotic core plaque components $<10 \%$;

- Pathological intimal thickening (PIT): fibrofatty plaque component $>10 \%$, and confluent necrotic core or calcium amounting to $<10 \%$ of the total plaque cross-sectional area;

- Fibroatheroma (FA): a confluent necrotic core comprising > $10 \%$ of the total plaque cross-sectional area;

- Fibrocalcific (FCa): a confluent area of calcium comprising $>10 \%$ of the total plaque cross-sectional area, with necrotic core and fibrofatty plaque each $<10 \%$ of the total plaque cross-sectional area;

- Thin-cap fibroatheroma (TCFA): high-risk plaque with a necrotic core comprising $>10 \%$ of the total plaque cross-sectional area and confluent with the lumen.

\section{Results}

The final study group consisted of 30 patients who underwent both DSCT and IVUS-VH examinations. The DSCT
Table 3. Number of cases of atherosclerotic plaque types in DSCT according to the de Weert classification

\begin{tabular}{|l|c|}
\hline De Weert classification & Number of cases \\
\hline Smooth wall surface - type 1 & 13 \\
Discreet wall irregularity - type 2 & 10 \\
Wall ulceration - type 3 & 7 \\
Total & 30 \\
\hline
\end{tabular}

results were classified according to the de Weert classification. Thirteen cases (43\%) were characterized as having a smooth wall surface, 10 cases (33\%) had discreet wall irregularities, and in 7 cases (23\%) incursions of contrast were observed, indicating the presence of ulceration (Table 3).

In the IVUS-VH examinations, 4 out of 30 cases (13\%) were identified as AIT, $4(13 \%)$ as PIT, $6(20 \%)$ as FA, $6(20 \%)$ as FCa and 10 (33\%) as TCFA (Table 4).

Table 4. Number of cases of each atherosclerotic plaque type according to plaque morphology characteristics (IVUS-VH)

\begin{tabular}{|l|c|}
\hline Plaque type & Number of cases \\
\hline AIT & 4 \\
PIT & 4 \\
FA & 6 \\
FCa & 6 \\
TCFA & 10 \\
\hline
\end{tabular}

The results of the de Weert classification of the DSCT results were compared with the results of plaque morphology classification obtained using IVUS-VH (Fig. 1). It was found that all the patients with confirmed presence of wall ulceration in DSCT were characterized as having high-risk plaque (TCFA) in IVUS-VH. Patients with discreet wall irregularities in DSCT formed a heterogeneous group in IVUS-VH: 6 lesions with a necrotic core (3 FA and 3 TCFA) and 4 lesions without a necrotic core (2 FCA, 1 PIT, 1 AIT). Among the 13 patients with smooth wall surfaces in DSCT, IVUS-VH showed 4 FCA lesions, 3 PIT lesions, 3 AIT lesions and 3 FA lesions (Table 5).

\section{Discussion}

The degree of ICA stenosis is an important predicator of ischemic cerebrovascular events and is universally used in therapeutic decision-making. Patients with both symptomatic and asymptomatic ICA stenosis above 70\% are considered candidates for endarterectomy or stent 


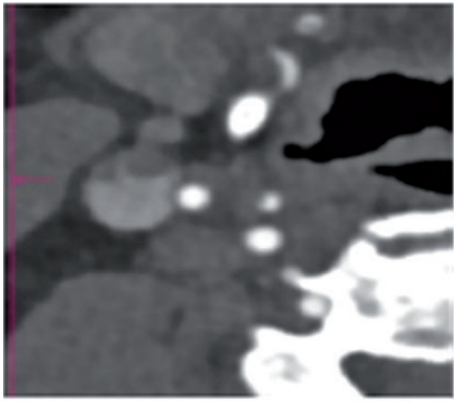

Type 1 - smooth wall surface

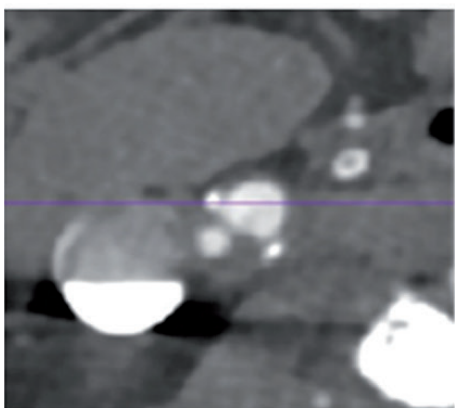

Type 2 - discreet wall irregularity

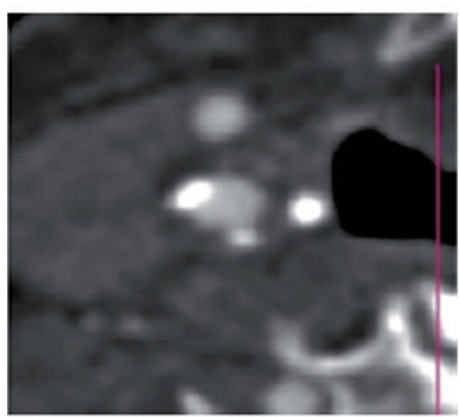

Type 2 - discreet wall irregularity

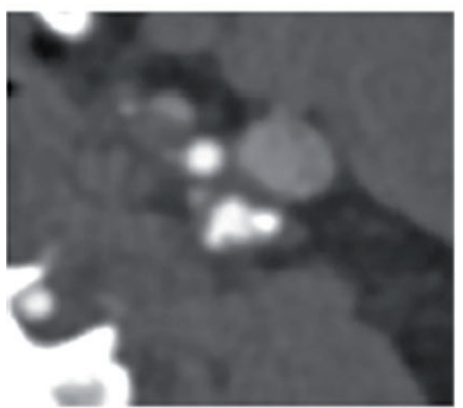

Type 3 - incursions of contrast

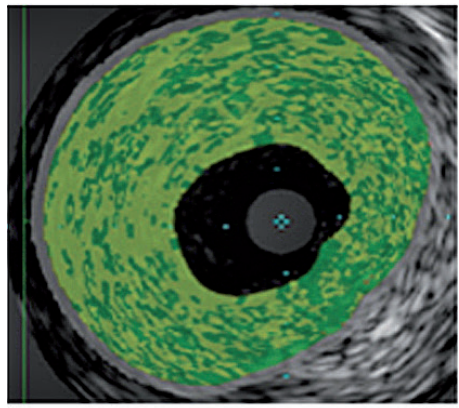

Adaptive intimal thickening Pathological intimal thickening Fibroatheroma

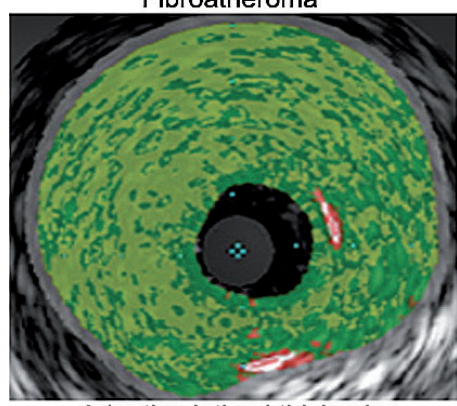

Adaptive intimal thickening Pathological intimal thickening

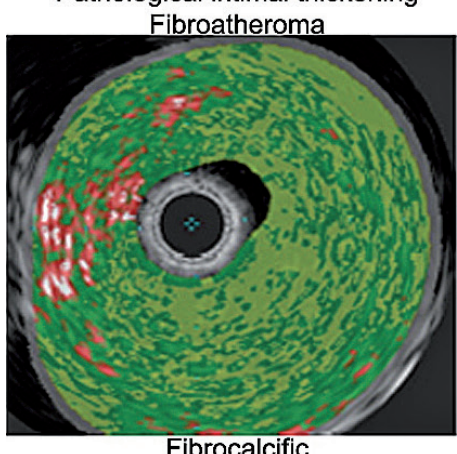

Fibrocalcific

Thin-cap fibroatheroma

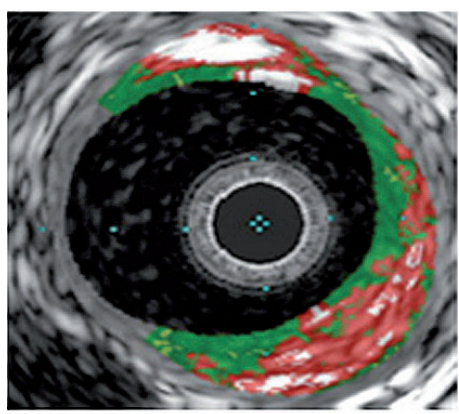

Thin-cap fibroatheroma
Fig. 1. DSCT with the de Weert classification (left) compared with IVUS-VH plaque morphology classification (right) placement. $^{2-4}$ However, it has been noted that advanced atherosclerosis may be found in non-stenotic artery segments due to the phenomenon of artery remodeling. ${ }^{17,18}$ Therefore, the morphological features of atherosclerotic plaque have been suggested as a complement to luminal narrowing measurements in stroke risk assessment. ${ }^{9-11,19}$ Several studies have demonstrated that the presence of a specific vulnerable plaque type (with a large lipid core and a thin fibrous cap) can be used as an independent risk predictor of ischemic cerebrovascular events. ${ }^{7,8}$ The clinical consequences of plaque ulceration have been clearly demonstrated in several studies, including NASCET. ${ }^{3,20,21}$

To develop the NASCET and ECST scales for carotid pathology detection, digital subtraction angiography (DSA) was used, which made this method the gold standard in ICA stenosis evaluation. Due to its availability and low cost, ultrasound imaging (US) is currently the most widely used diagnostic method for detecting and assessing the extent of carotid atherosclerosis. However, there is no universally accepted method that provides a satisfactory amount of information in vivo about ICA plaque morphology. In their study, Saba et al. compared the diagnostic efficacy of multidetector computed tomography angiography (MDCTA) and US with echo color Doppler in patients with carotid plaque complicated by ulceration. ${ }^{22}$ When the data from MDCTA and US imaging were set side by side with surgical specimens taken during carotid endarterectomy, it was demonstrated that MDCTA has very high sensitivity (93.75\%) and specificity (98.59\%) in detecting plaque ulcerations of the carotid artery.

To explore the potential of $\mathrm{CT}$ in assessing plaque morphology, the authors needed a well-established reference method. The choice fell on IVUS-derived virtual histology (IVUS-VH), which is used for the detection and quantification of non-stenotic atheroma in coronary arteries. ${ }^{13}$ The IVUS-VH imaging protocol uses advanced radiofrequency analysis of ultrasound signals, enhancing the image with colors in correlation to different amplitudes and frequencies. This makes it possible to recognize vulnerable plaque by assessing their structure and composition, differentiating four primary plaque components: fibrotic tissue, calcifications and lipid or necrotic cores. IVUS-VH findings demonstrate a good correlation with histopathology results. ${ }^{23,24}$

Table 5. Number of different morphological types of atherosclerotic plaque (IVUS-VH) correlated with de Weert classification types (DSCT)

\begin{tabular}{|c|c|c|c|c|c|c|}
\hline de Weert type & Number of patients & TCFa & $\mathrm{FCa}$ & PIT & FA & AIT \\
\hline 1 & 13 & 0 & 4 & 3 & 3 & 3 \\
\hline 2 & 10 & 3 & 2 & 1 & 3 & 1 \\
\hline 3 & 7 & 7 & 0 & 0 & 0 & 0 \\
\hline Total & 30 & 10 & 6 & 4 & 6 & 4 \\
\hline
\end{tabular}


As already noted, IVUS-VH is used as a reference standard in evaluating other methods of imaging atherosclerotic plaque morphology in coronary arteries. ${ }^{25}$ However, the authors did not find any publications concerning the usefulness of IVUS-VH in evaluating DSCT results in ICA plaque morphology assessments. Until now, studies have focused on determining a correlation between CT results and histopathological findings gathered during endarterectomies. Fragmentation or damage to the sample - which often occurs during the procedure - makes it impossible to pinpoint the exact location of the histopathological specimen with respect to $\mathrm{CT}$ images. For this reason, a correlation can be rendered only with regard to general morphological composition. ${ }^{26}$

In their study, de Weert et al. noted that they had difficulties with the identification of ulceration in highly stenotic vessels. ${ }^{12}$ The calcification burden rises with severe stenosis. With MDCTA, differentiating between contrast enhancement and strongly calcified lesions was often impossible, which hampered the assessment of plaque morphology. The current authors did not encounter such difficulties in distinguishing contrast material from calcifications, thanks to the availability of a new dual-source multidetector CT system (DSCT).

Introduced in 2009, DSCT is equipped with 2 X-ray tubes that can be operated at different $\mathrm{kV}$ settings (an $80 \mathrm{kV}$ lamp paired with a $120 \mathrm{kV}$ or $140 \mathrm{kV}$ lamp) and different prefiltration. Due to the simultaneous use of two X-ray sources, it is possible to obtain different radiation absorption coefficients (in Hounsfield units) for any given tissue. This allows better tissue differentiation and advanced image post-processing. DSCT permits reliable subtraction of calcified structures such as bones and plaque; therefore, it makes evaluation of the grade of stenosis and plaque morphology possible, even in highly stenotic vessels. ${ }^{27}$ Dual-energy acquisition and more detector rows make examination times shorter and reduce the radiation absorbed by patients. Shorter scan time also means less contrast (50-60 mL). Therefore, DSCT enhanced with plaque and bone subtraction algorithms (PBS) has the potential to identify patients with vulnerable plaque better than conventional $\mathrm{CT}$.

Work on CT number-based plaque characterization with the use of absolute Hounsfield unit (HU) ranges to separate plaque components began with the advent of new software. ${ }^{25}$ Unified HU-based atherosclerotic plaque characterization criteria have not yet been created. Metaanalyses indicate that this approach is limited due to its dependence on factors such as lumen contrast opacification, the degree of artery stenosis, image noise, partial volume effects, undershooting artifacts and tube voltage. ${ }^{28}$ Moreover, existing classifications do not work well for identifying lipid-rich necrotic cores and hemorrhages, because the range of densities associated with these components is similar to those for connective tissue. This overlap severely limits the reliability of individual pixel Hounsfield readings on key components of vulnerable plaque. ${ }^{29}$
At present, there is no satisfactory method for direct assessment of plaque morphology in CT. ${ }^{30,31}$ This is the reason the current authors considered adapting de Weert classification for DSCT data analysis, which uses plaque surface morphology assessment. The indirect approach is, in the current authors' opinion, a feasible and reliable tool for clinical use. Nevertheless, the distinction between atherosclerotic plaque morphology and plaque surface morphology has to be recognized.

In the present study, only a partial correlation was found between the results of DSCT with the de Weert classification and the results of IVUS-VH with plaque morphology classification. Nevertheless, the authors find this outcome clinically important. The data show that all the lesions with surplus contrast (type 3 in the de Weert classification) had the morphological features of TCFA, i.e. the presence of a necrotic component $>10 \%$. According to the plaque morphology classification, TCFA is considered high-risk plaque with a propensity for rupturing. In fact, TCFA bears a strong morphological resemblance to plaque rupture even though it exhibits a smaller necrotic core, a thin fibrous cap infiltrated by a smaller number of macrophages, and a lower degree of calcification. ${ }^{5}$

This study has some limitations. Firstly, the study group consisted of only 30 patients. Secondly, IVUS-VH has its own intrinsic limitations as a method of reference. In the case of strong remodeling, the transducer penetration depth $(3-6 \mathrm{~mm})$ may be insufficient to evaluate a complete cross-section of the ICA wall. The IVUS transducer itself is more suitable for coronary arteries, and the wider lumen of the carotid artery may cause non-axial settlement that results in an elliptic rather than orthoradial scan of an artery. However, that would more likely cause a misevaluation of the degree of stenosis rather than an incorrect assessment of plaque morphology.

DSCT with the de Weert classification of plaque surface morphology makes the reliable detection of ulcerations possible. The present study demonstrated that surplus the contrast agent in DSCT (indicating the presence of ulceration) correlates with the presence of unstable TCFA plaque in IVUS-VH. The benefits of surgical or endovascular intervention in symptomatic patients who do not meet the criteria for severe stenosis, but in whom the presence of ulcerated plaque has been identified, needs to be demonstrated in larger prospective studies. Nevertheless, the authors are confident that diagnostic algorithms in patients with cerebrovascular ischemic risk should be enriched with an assessment of atherosclerotic plaque morphology. Due to the unavailability of a satisfactory method that allows for direct assessment of plaque morphology in DSCT, an indirect approach using DSCT with de Weert classification could offer a significant new technique to improve current imaging protocols. 


\section{References}

1. Mozaffarian D, et al. Executive summary: Heart disease and stroke statistics-2016 update: A report from the American Heart Association. Circulation. 2016;133: 447-454.

2. Rothwell PM, Eliasziw M, Gutnikov SA, et al. Analysis of pooled data from the randomized controlled trials of endarterectomy for symptomatic carotid stenosis. Lancet. 2003;11:107-116.

3. North American Symptomatic Carotid Endarterectomy Trial Collaborators. Beneficial effect of carotid endarterectomy in symptomatic patients high with grade stenosis: N EnglJ Med. 1991; 15:445-453.

4. European Carotid Surgery Trialists' Collaborative Group MRC European Carotid Surgery Trial: Interim results for symptomatic patients with severe (70\%-99\%) or with mild (0\%-29\%) carotid stenosis. Lancet. 1991;25:1235-1243.

5. Narula J, Nakano M, Virmani R, et al. Histopathologic characteristics of atherosclerotic coronary disease and implications of the findings for the invasive and noninvasive detection of vulnerable plaques. J Am Coll Cardiol. 2013;12:1041-1051.

6. Naghavi M, Libby P, Falk E, et al. From vulnerable plaque to vulnerable patient: A call for new definitions and risk assessment strategies: Part I. Circulation. 2003;14:1772-1778.

7. Gupta A, Baradaran H, Schweitzer AD, et al. Carotid plaque MRI and stroke risk: A systematic review and meta-analysis. Stroke. 2013;44:3071-3077.

8. Howard DP, van Lammeren GW, Rothwell PM, et al. Symptomatic carotid atherosclerotic disease: Correlations between plaque composition and ipsilateral stroke risk. Stroke 2015;46(1):182-189.

9. Huibers A, de Borst GJ, Wan S, et al. Non-invasive carotid artery imaging to identify the vulnerable plaque: Current status and future goals. Eur J Vasc Endovasc Surg. 2015;50:563-572.

10. Brinjikji W, Huston J, Rabinstein AA, Kim GM, Lerman A, Lanzino G. Contemporary carotid imaging: from degree of stenosis to plaque vulnerability. J Neurosurg. 2016;124(1):27-42.

11. Truijman MT, Kooi ME, van Dijk AC, et al. Plaque at RISK (PARISK): Prospective multicenter study to improve diagnosis of high-risk carotid plaques. Int J Stroke. 2014; 9:747-754.

12. de Weert TT, Cretier S, Groen HC, et al. Atherosclerotic plaque surface morphology in the carotid bifurcation assessed with multidetector computed tomography angiography. Stroke. 2009;40(4): 1334-1340.

13. Konig A, Margolis MP, Virmani R, Holmes D, Klauss V. Technology insight: In vivo coronary plaque classification by intravascular ultrasonography radiofrequency analysis. Nat Clin Pract CardiovasC Med. 2008;5(4):219-229.

14. Anders Persson, Protocol Dual Energy Head and Neck CTA, Dual Source CT community http://www.dsct.com/index.php/protocol-dual-energy-head-and-neck-cta/ Published April 8, 2008, Accesed January 2, 2011

15. Lanzer $P$, Mastering endovascular techniques: $A$ guide to excellence, Philadelphia 2006, $1^{\text {st }}$ ed. Lippincott Williams \& Wilkins, 85-86.

16. Stary HC, Chandler AB, Dinsmore RE, et al. A definition of advanced types of atherosclerotic lesions and a histological classification of atherosclerosis. A report from the Committee on Vascular Lesions of the Council on Arteriosclerosis, American Heart Association. Circulation. 1995;1;92(5):1355-1374.
17. Mintz GS, Painter JA, Pichard AD, et al. Atherosclerosis in angiographically "normal" coronary artery reference segments: an intravascular ultrasound study with clinical correlations. J Am Coll Cardiol. 1995;25(7):1479-1485.

18. Coutinho JM, Derkatch S, Potvin AR, et al. Nonstenotic carotid plaque on CT angiography in patients with cryptogenic stroke. Neurology. 2016;16:665-672.

19. Rothwell PM, Gibson R, Warlow CP. Interrelation between plaque surface morphology and degree of stenosis on carotid angiograms and the risk of ischemic stroke in patients with symptomatic carotid stenosis. On behalf of the European Carotid Surgery Trialists' Collaborative Group. Stroke. Stroke. 2000;31(3):615-621.

20. Park AE, McCarthy WJ, Pearce WH, Matsumura JS, Yao JS. Carotid plaque morphology correlates with presenting symptomatology. J Vasc Surg. 1998;27(5):872-878.

21. Lovett JK, Gallagher PJ, Hands LJ, Walton J, Rothwell PM. Histological correlates of carotid plaque surface morphology on lumen contrast imaging. Circulation. 2004;12:2190-2197.

22. Saba L, Caddeoc G, Sanfilippob R, Montiscib R, Mallarinia G. CT and ultrasound in the study of ulcerated carotid plaque compared with surgical results: Potentialities and advantages of multidetector row CT angiography. AJNR Am J Neuroradiol. 2007;28(6):1061-1066.

23. Nasu K, Tsuchikane E, Katoh O, et al. Accuracy of in vivo coronary plaque morphology assessment: A validation study of in vivo virtual histology compared with in vitro histopathology. J Am Coll Cardiol. 2006;20:2405-2412.

24. Nair A, Kuban BD, Tuzcu EM, Schoenhagen P, Nissen SE, Vince DG. Coronary plaque classification with intravascular ultrasound radiofrequency data analysis. Circulation. 2002;22:2200-2206.

25. Springer I, Dewey M. Comparison of multislice computed tomography with intravascular ultrasound for detection and characterization of coronary artery plaques: A systematic review. Eur J Radiol. 2009;71:275-282.

26. Das M, Braunschweig $T$, Mühlenbruch $G$, et al. Carotid plaque analysis: Comparison of dualsource computed tomography (CT) findings and histopathological correlation. Eur J Vasc Endovasc Surg. 2009;38(1):14-19.

27. Flohr TG, McCollough $\mathrm{CH}$, Bruder $\mathrm{H}$ et al. First performance evaluation of a dual-source CT (DSCT) system. Eur Radiol. 2006;16(2):256268.

28. Kristanto W, van Ooijen PM, Jansenvan der Weide MC, Vliegenthart $\mathrm{R}$, Oudkerk M. A meta analysis and hierarchical classification of $\mathrm{HU}$-based atherosclerotic plaque characterization criteria. PLoS One. 2013;3:e73460.

29. Wintermark M, Jawadi SS, Rapp JH, et al. High-resolution CT imaging of carotid artery atherosclerotic plaques. AJNR Am J Neuroradiol. 2008;29(5):875-882.

30. Obaid DR, Calvert PA, Gopalan D, et al. Dual-energy computed tomography imaging to determine atherosclerotic plaque composition: A prospective study with tissue validation. J Cardiovasc Comput Tomogr. 2014;8(3):230-237.

31. Hetterich $\mathrm{H}$, Webber $\mathrm{N}$, Willner $\mathrm{M}$, et al. AHA classification of coronary and carotid atherosclerotic plaques by grating-based phase-contrast computed tomography. Eur Radiol. 2016;26(9):3223-3233. 